\title{
ESTRATEGIAS PARA LA RESTAURACIÓN DE PAISAJES FORESTALES EXPERIENCIAS EN MISIONES, ARGENTINA
}

\author{
Florencia Montagnini ${ }^{1}$ Beatriz Eibl2 Roberto Fernández ${ }^{2.3}$, Matthew Brewer ${ }^{1}$
}

\section{RESUMEN}

La restauración de ecosistemas forestales degradados se puede beneficiar con la implementación de estrategias diversificadas para generar ingresos. Estas estrategias disminuyen el riesgo y aumentan la factibilidad de adopción por parte de los agricultores. Las estrategias deben estar adaptadas al tipo de degradación que se encuentre, definida por el grado de deterioro quimico y físico de los suelos, invasión de especies agresivas. empobrecimiento de especies, y dificultades en el proceso de sucesión forestal natural. En la provincia de Misiones, Argentina, los proyectos de reforestación y el enriquecimiento forestal de bosques degradados empleando especies nativas, son estrategias viables para mantener la biodiversidad y recuperar el ecosistema forestal.

La Provincia de Misiones tiene menos del 1\% de la superficie total del pais, pero alberga casi el $40 \%$ de la biodiversidad y produce más del $70 \%$ de la madera. Misiones ha tenido cambios de uso de la tierra debido al corte de bosques para el establecimiento de plantaciones industriales y cultivos comerciales. Usos de la tierra alternativos son necesarios debido a la agricultura migratoria y al uso de técnicas inadecuadas del manejo de suelos que ha llevado a la degradación de suelos y al abandono de tierras.

En 1990-1991 se ensayó una serie de plantaciones mixtas y sistemas agroforestales con yerba mate (llex paraguariensis) en sitios con condiciones contrastantes de degradación. Enterolobium contortisiliquum y Astronium balansae tuvieron el mejor crecimiento en suelos pobres. Balfourodendron riedelianum, una especie valiosa en la región tuvo comportamiento excelente en suelos fértiles y con manejo adecuado. La asociación de árboles con la yerba mate fue aparentemente apropiada, ya que las cosechas de yerba mate representan un retorno de la inversión a corto plazo.

En experimentos de enriquecimiento de bosques degradados, en la Reserva Guarani, a los 13 años las mejores especies fueron Ocotea puberula, Bastardiopsis densiflora, y Cordia trichotoma. Estas especies también tienen efectos beneficiosos sobre los suelos de los bosques degradados. La incorporación de especies con cosecha más temprana tal como la palma, Euterpe edulis, puede acelerar el retorno de la inversión.

1-Yale University School of Forestry and Environmental Studies, 370 Prospect Street, New Haven, CT 06511, USA. email: florencia.montagnini@yale.edu

2-Facultad de Ciencias Forestales, Universidad Nacional de Misiones, (3382) Eldorado, Misiones, Argentina, email: beibl@facfor.unam.edu.ar

2-3 INTA Monte Carlo,(3384) Monte Carlo, Misiones. Argentina, email: rernandez@montecarlo.inta gov.ar 
Resultados de estas investigaciones se están utilizando para estimular la plantación de árboles en proyectos comunitarios, tanto en sistemas agroforestales o de plantaciones a cielo abierto como de enriquecimiento de bosques degradados.

Palabras clave: Forestación Áreas Degradadas, Enriquecimiento Bosques Degradados,

\section{STRATEGIES FOR RESTORATION OF FOREST LANDSCAPES. EXPERIENCES FROM MISIONES, ARGENTINA}

\section{SUMMARY}

The successful restoration of degraded forest ecosystems can benefit from the implementation of diversified income generation strategies. These strategies may reduce risk and allow for more ready adoption of restoration projects by local farmers. In the Province of Misiones, Argentina, large-scale native-species reforestation projects, coupled with enrichment plantings in degraded forests, are plausible strategies for maintaining biodiversity and forest health.

The Province of Misiones covers less than $1 \%$ of the total area of the country, but harbors almost $40 \%$ of the biodiversity and produces over $70 \%$ of the timber. Misiones had substantial land use changes due to forest clearing for industrial plantations and commercial crops. Alternative land uses are necessary due to shifting agriculture and use of inadequate soil techniques leading to soil degradation and land abandonment.

In 1990-1991 a set of experiments was put in place to test mixed plantations and agroforestry with yerba mate (Ilex paraguariensis) on sites with contrasting soil degradation. Enterolobium contortisiliquum and Astronium balansae showed the best growth on poor soils. Balfourodendron riedelianum, a valued tree in this region had excellent performance on fertile soils and with adequate management. The association of trees with yerba mate appeared appropriate, since yerba mate harvests represent a short-term return on investment.

Enrichment experiments were established in 1988-1990 in degraded forests. In the Guarani Reserve, the best species at thirteen years were Ocotea puberula, Bastardiopsis densiflora, and Cordia trichotoma. These species also have beneficial effects on soils, increasing soil nitrogen and base cation concentrations and thus improving conditions of degraded forests. The incorporation of species with shorter harvest age such as the Euterpe edulis palm can accelerate investment returns.

Results of this research are used to stimulate tree planting in community forestry projects, both in open plantation and in enrichment planting of degraded secondary forest.

Key words: Degraded Lands Afforestation, Degraded Forests Enrichment. 


\section{INTRODUCCION}

La Provincia de Misiones, NE de Argentina (25 LS, $\left.45^{\circ} \mathrm{LO}\right)$, cubre una superficie de $30.000 \mathrm{~km}^{2}$, que representa aproximadamente el $1 \%$ de la superficie total del pais (Figura $\mathrm{N}^{\circ} 1$ ). sin embargo el 70 de la madera de aserrio y más del $85 \%$ de la madera compensada del pais proviene de esta Provincia (Gobierno de la Provincia de Misiones, 2003).

El uso predominante de la tierra en Misiones está dado por plantaciones forestales con especies exóticas (Pinus spp., Eucalyptus spp., Melia azederach L., Toona ciliata M Roem var Australis, Paulownia spp., Grevillea robusta A.), plantaciones forestales de árboles nativos como Araucaria angustifolia (Bert) Otto Kuntze, cultivos agricolas perennes como yerba mate (Ilex paraguariensis), citrus (Citrus spp.), y cultivos anuales de maiz, mandioca y soja, tabaco. ganaderia y aprovechamientos comerciales en bosques nativos (Montagnini et al., 2006a. 2006b).

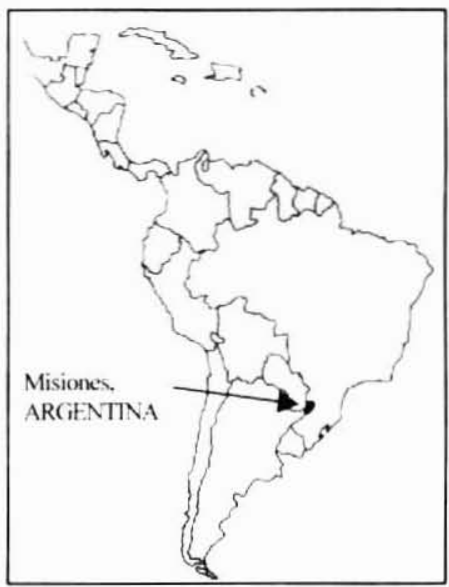

Figura $\mathrm{N}^{\circ} 1$

\section{LOCALIZACIÓN DE LA PROVINCIA DE MISIONES EN EL NE DE ARGENTINA}

Las superficies de terrenos abandonados luego de ser utilizados durante décadas por intensa actividad agricola, generan extensiones importantes de suelo degradado y compactado. que para la Provincia de Misiones significan aproximadamente 600 mil hectáreas (Ministerio de Ecologia y Recursos Naturales Renovables y Turismo, 2003).

En los bosques de Misiones, el sistema de diámetro minimo de corta, que oscila entre 40 y $55 \mathrm{~cm}$ de diámetro a la altura del pecho (DAP) segủn las especies, tiene una serie de efectos indeseables. Por ejemplo, se extraen todos los individuos adultos que servirian como semilleros para las generaciones futuras. Esto favorece el crecimiento de especies heliófilas invasoras, tales como bambúseas en el sotobosque, debido a la amplia apertura del dosel realizada con la extracción, lo cual tiende a impedir la regeneración de especies arbóreas umbrófilas (Montagnini et al., 1997, 1998). 
Entre las alternativas planteadas para la recuperación de los bosques degradados y secundarios se plantea la recomposición de la masa forestal mediante técnicas de enriquecimiento con especies arbóreas. El enriquecimiento es una opción para cuando la regeneración natural es insuficiente y las caracteristicas del suelo son poco indicadas para otros usos. El enriquecimiento consiste en la introducción de especies arbóreas valiosas en bosques degradados sin eliminar los árboles presentes. El enriquecimiento puede incluir árboles frutales o de otros usos alternativos.

La restauración de ecosistemas forestales degradados se puede beneficiar con la implementación de estrategias diversificadas para generar ingresos. Estas estrategias disminuyen el riesgo y aumenta la factibilidad de adopción por parte de los agricultores. Las estrategias deben estar adaptadas al tipo de degradación que se encuentre, definida por el grado de deterioro quimico y físico de los suelos, invasión de especies agresivas, empobrecimiento de especies, y dificultades en el proceso de sucesión forestal natural.

En Misiones, los proyectos de reforestación y el enriquecimiento forestal de bosques degradados empleando especies nativas, son estrategias viables para mantener la biodiversidad y recuperar el ecosistema forestal.

El conocimiento de especies nativas que al mismo tiempo son económicamente valiosas y contribuyen a la recuperación de la fertilidad de los suelos es especialmente relevante porque, además de la eventual mejora de las condiciones de los suelos, la plantación de estas especies provee fuentes de ingreso a los agricultores. Las especies nativas además de su madera de alto valor comercial pueden estar mejor adaptadas al ecosistema local. Muchas especies que tradicionalmente son extraidas del bosque natural para fines maderables actualmente están en peligro de extinción, por este motivo las plantaciones comerciales con las mismas especies podrian reemplazar esta demanda.

En la Provincia de Misiones son pocas las experiencias de plantaciones con especies forestales nativas a cielo abierto. La Facultad de Ciencias Forestales de la Universidad Nacional de Misiones (UNAM), en colaboración con la Universidad de Yale (USA), mantiene desde 1989 investigaciones sobre la ecologia de especies forestales nativas de la selva subtropical misionera, y su utilización en sistemas de producción (Eibl y Montagnini, 1998).

En la Provincia de Misiones las experiencias de enriquecimiento de bosques degradados datan de la década de los 1960. Mangieri (1965) y Cozzo (1969) recomiendan el uso de Cedrela fissilis, Cordia trichotoma, Tabebuia ipe y Myrocarpus frondosus para su implantación bajo cubierta, dado que estas especies son sensibles a las heladas, lo cual dificulta su plantación a cielo abierto. Gartland (1974) describe un ensayo ubicado en Puerto Península, al NE de la Provincia, donde se utilizó Melia azedarach var. gigantea, Balfourodendron riedelianum y Cordia trichotoma bajo cubierta de bosque secundario. A los dos años estas especies superaron al dosel del bosque secundario de 4 metros de altura. Sánchez et al. (1993) al N de la provincia ensayaron un grupo de especies nativas y exóticas, dando recomendaciones especificas de manejo, estimativas de turnos de cortas y evaluación económica de las prácticas.

En el presente trabajo se informa sobre el comportamiento de especies forestales 
nativas de potencial productivo con posible adaptación a sitios degradados y se describen ensayos de enriquecimiento con especies arbóreas maderables nativas, realizados en bosques explotado por diámetro mínimo, en los cuales la situación de la masa remanente no justificaba aprovechamientos futuros a mediano y largo plazo.

\section{OBJETIVOS}

Evaluar la adaptación y crecimiento de especies en sitios degradados en diferentes condiciones de plantación como cultivo puro o mixto y en combinaciones agroforestales, y evaluar plantaciones de enriquecimiento con especies maderables nativas en bosques naturales empobrecidos por explotación.

\section{REFORESTACION DE AREAS DEGRADADAS CON ARBOLES NATIVOS}

\section{Materiales y Métodos}

\section{- Sitios de Estudio}

Las investigaciones se realizaron en tres sitios que habian sostenido diferentes usos de la tierra y presentaban, al momento de comenzar los ensayos, diferentes grados de degradación. El clima se clasifica como Cfa en el sistema de Köppen, que corresponde a un clima subtropical húmedo sin estación seca, con precipitación media anual de $1700 \mathrm{~mm}$, distribuida equitativamente a lo largo del año, y media anual de temperatura de $21^{\circ} \mathrm{C}$, con máximas absolutas de $39^{\circ} \mathrm{C}$ (Enero) y minimas absolutas de $-6^{\circ} \mathrm{C}$ (Julio) (Eibl et al., 2001). agricolas.

El sitio más degradado (Sitio 1) fue un terreno utilizado durante 40 años para cultivos

El Sitio 2 se encontraba medianamente degradado, consistiendo en un campo cubierto por pastos invasores (Andropogum spp., Pennisetum spp.). Este sitio había sido abandonado luego de aproximadamente 20 años de agricultura mecanizada intensiva.

El Sitio 3 fue el de menor degradación. Su uso anterior fue una plantación de tung (Aleuritis fordii Forst.) por aproximadamente 20 años, establecida luego de aprovechar el bosque nativo.

Al momento de la instalación de los ensayos se realizaron relevamientos de suelo, con muestras al azar de las diferentes parcelas y una calicata para evaluar y describir el perfil del sitio. (Cuadro $\mathrm{N}^{\circ} 1$ ). 


\begin{tabular}{|c|c|c|c|c|c|c|c|c|c|c|}
\hline \multirow[b]{3}{*}{ Sitio } & \multicolumn{10}{|c|}{$\begin{array}{c}\text { Cuadro } N^{\circ} 1 \\
\text { CARACTERISTICAS QUIMICAS DE LOS SUELOS } \\
\text { ANTES DE REALIZAR LAS PLANTACIONES PARA LOS TRES SITIOS }\end{array}$} \\
\hline & \multirow{2}{*}{$\begin{array}{l}\text { Espesor } \\
(\mathrm{cm})\end{array}$} & \multirow{2}{*}{$\begin{array}{l}\text { MO } \\
(\%)\end{array}$} & \multirow{2}{*}{$\begin{array}{l}\text { Nt } \\
(\%)\end{array}$} & \multirow{2}{*}{$\begin{array}{c}P \\
(p p m)\end{array}$} & \multirow{2}{*}{$\begin{array}{l}\mathrm{pH} \\
(\mathrm{en} \\
\left.\mathrm{H}_{2} \mathrm{O}\right)\end{array}$} & $\mathrm{Ca}^{2+}$ & $\mathrm{Mg}^{2+}$ & $\mathbf{K}^{+}$ & \multirow{2}{*}{$\begin{array}{l}\text { Suma de } \\
\text { bases } \\
\left(\mathrm{cmol}^{+} / \mathrm{kg}\right)\end{array}$} & \multirow{2}{*}{$\begin{array}{c}\mathrm{CIC} \\
\left(\mathrm{cmol}^{+} / \mathrm{kg}\right)\end{array}$} \\
\hline & & & & & & \multicolumn{3}{|c|}{$\left(\mathrm{cmol}^{+} / \mathrm{kg}\right)$} & & \\
\hline \multirow{2}{*}{ Sitio 1} & $0-10$ & 2,1 & 0,09 & 1,5 & 5,2 & 4,3 & 1,1 & 0,20 & 5,6 & 7,7 \\
\hline & $10-30$ & 0,9 & 0,07 & 1,0 & 5,1 & 3,6 & 1,0 & 0,09 & 4,7 & 7,0 \\
\hline \multirow{2}{*}{ Sitio 2} & $0-10$ & 2,6 & 0,24 & 3,2 & 5,2 & 5,8 & 1,5 & 0,29 & 7,6 & 10,7 \\
\hline & $10-30$ & 1,4 & 0,12 & 1,3 & 4,7 & 4,2 & 0,9 & 0,11 & 5,2 & 8,7 \\
\hline \multirow{2}{*}{ Sitio 3} & $0-10$ & 3,9 & 0,31 & 5,4 & 5,4 & 8,6 & 2,3 & 0,41 & 11,3 & 19,6 \\
\hline & $10-30$ & 2,3 & 0,20 & 4,4 & 4,6 & 6,4 & 1,3 & 0,21 & 7,9 & 12,8 \\
\hline
\end{tabular}

\section{- Especies Ensayadas}

Las especies ensayadas fueron:

Enterolobium contortisiliquum (Vellozo) Morong Lonchocarpus muehlbergianum Hassler Astronium balansae Engl.

Peltophorum dubium (Sprengler) Taubert Balfourodendron riedelianum (Engler) Engler Tabebuia heptaphylla (Vellozo) Toledo
Fabaceae

Fabaceae

Anacardiaceae

Fabaceae

Rutaceae

Bignoniaceae timbó colorado rabo molle urunday cañafistola guatambú blanco lapacho negro

Estas especies fueron seleccionadas según experiencias anteriores por su potencial para el crecimiento a cielo abierto y sus posibles impactos positivos sobre los suelos (Montagnini et al., 1995; Fernández et al., 1997; Eibl y Montagnini, 1998), asi como por su valor maderable. Entre estas especies dos están referenciadas como fijadoras de nitrógeno (timbó colorado y rabo molle).

\section{- Diseño Experimental} tratamiento.

Sitio 1: Cuatro bloques al azar con seis parcelas cada una, con cuatro repeticiones por

Tratamiento 1: Enterolobium contortisiliquum (timbó colorado), fecha de plantación agosto de 1990.

Tratamiento 2: Peltophorum dubium (caña fistola), agosto de 1995.

Tratamiento 3: Astronium balansae (urunday), agosto de 1993. Estas dos últimas especies reemplazaron a Bastardiopsis densiflora (Hooker\&Arnott) Hassler (Ioro blanco) 
y a Balfourodendron riedelianum, las cuales no se adaptaron al sitio y fueron descartadas

Tratamiento 4: Lonchocarpus muehlbergianum (rabo molle), agosto de 1990.

Tratamiento 5: Mezcla de las cuatro especies intercaladas (timbó colorado, caña fistola. urunday y rabo molle).

Tratamiento 6: Testigo con pasto natural (sin árboles).

Los tamaños de las parcelas fueron de $18 \times 18 \mathrm{~m}$ y las distancias de plantación de los ejemplares de $3 \times 3 \mathrm{~m}$.

Sitio 2 y Sitio 3: Los tratamientos forman parte de ensayos agroforestales con especies maderables nativas asociadas con yerba mate (Ilex paraguariensis Saint Hilaire (Aquifoliaceae) (Eibl et al., 1997, 1998, 2000). La fecha de plantación fue agosto de 1991 y agosto 1993 para Tabebuia heptaphylla. El diseño consistió de cuatro bloques con cuatro parcelas cada una y con cuatro repeticiones por tratamiento.

Tratamiento 1: Enterolobium contortisiliquum (timbó) plantados a $3 \mathrm{~m} \times 6 \mathrm{~m}$, ó 555 plantas por hectárea, en lineas alternadas con yerba.

Tratamiento 2: Balfourodendron riedelianum (guatambú) plantado a $3 \mathrm{~m} \times 6 \mathrm{~m}$, ó 555 plantas por hectárea, en lineas alternadas con yerba.

Tratamiento 3: Balfourodendron riedelianum $(6 \mathrm{~m} \times 6 \mathrm{~m})$ y $\mathrm{E}$. contortisiliquum $(6 \mathrm{~m} \times 6$ $\mathrm{m})$. Estos se dispusieron en lineas mixtas alternadas con lineas de lex paraguariensis.

Tratamiento 4: Testigo, lex paraguariensis sola como cultivada tradicionalmente en la zona ( $3 \mathrm{~m} \times 1,5 \mathrm{~m}, 2222$ plantas por hectárea). Además en el Sitio 2, se incluyó Tabebuia heptaphylla (lapacho negro) $(3 \mathrm{~m} \times 6 \mathrm{~m})$ en lineas alternadas en plantaciones de yerba.

Cada parcela fue de $15 \times 15 \mathrm{~m}$. En los tres sitios se realizaron mediciones anuales de crecimiento en altura, diámetro a la altura del pecho, supervivencia y observaciones generales.

\section{- Resultados}

\section{Crecimientos y Supervivencias de las Especies para cada Sitio}

\section{Sitio 1}

La medición realizada en diciembre de 2004 (Cuadro $N^{\circ} 2$ ), cuando las especies tenian entre 9 y 14 años reveló que los mayores diámetros y alturas se encontraban en las parcelas de timbó (con 14 años) tanto para la situación pura como mixta. En segundo lugar le sigue el 
urunday (con 11 años) tanto para la situación pura como mixta. En tercer lugar, cañafístola con mayor diámetro y altura para las plantaciones mixtas y con una menor supervivencia. Rabo molle tuvo el menor crecimiento tanto en diámetro como altura. Las supervivencias fueron superiores en el caso de plantación mixta.

\section{Cuadro $\mathrm{N}^{\circ} 2$ \\ CRECIMIENTO EN DIÁMETRO, ALTURA Y SUPERVIVENCIA DE CUATRO ESPECIES FORESTALES NATIVAS EN CONDICIONES PURAS Y MIXTAS}

\begin{tabular}{|l|c|c|c|}
\hline ESPECIE & $\begin{array}{c}\text { DIAMETRO } \\
(\mathbf{c m})\end{array}$ & $\begin{array}{c}\text { ALTURA (m) } \\
\text { Enterolobium contortisiliquum puro }\end{array}$ & $\begin{array}{c}\text { SUPERVIVENCIA } \\
\text { (\%) }\end{array}$ \\
\hline Enterolobium contortisiliquum mixto & $25,4 \mathrm{a}$ & $11.82 \mathrm{a}$ & $46 \mathrm{c}$ \\
\hline Peltophorum dubium puro & $8,45 \mathrm{c}$ & $10,6 \mathrm{a}$ & $70 \mathrm{a}$ \\
\hline Peltophorum dubium mixto & $10,2 \mathrm{~b}$ & $9,6 \mathrm{~d}$ & $78 \mathrm{a}$ \\
\hline Astronium balansae puro & $10,2 \mathrm{~b}$ & $9,2 \mathrm{~d}$ & $56 \mathrm{c}$ \\
\hline Astronium balansae mixto & $10,9 \mathrm{~b}$ & $8,2 \mathrm{c}$ & $59 \mathrm{~b}$ \\
\hline Lonchocarpus muehlbergianum puro & $7,4 \mathrm{~d}$ & $4,4 \mathrm{e}$ & $78 \mathrm{a}$ \\
\hline Lonchocarpus muehlbergianum mixto & $2,8 \mathrm{e}$ & $2,7 \mathrm{f}$ & $48 \mathrm{c}$ \\
\hline
\end{tabular}

Sitio 1. Mediciones diciembre de 2004. Letras diferentes indican diferencias significativas entre grupos. (Fuente: Montagnini et al.. 2006b)

Ambas especies consideradas fijadoras de nitrógeno (timbó y rabo molle) presentaron las menores supervivencias, a pesar de que se realizaron reposiciones hasta el tercer año. El timbó requirió podas de corrección del fuste en forma anual y el rabo molle presentó crecimientos lentos. Para cañafistola no se han realizado reposiciones, y se realizaron solamente algunas podas de corrección del fuste ya que su crecimiento es marcadamente monopódico. Todas las especies debieron ser protegidas contra las heladas por uno a dos inviernos luego de plantadas, utilizándose para ello el material vegetal que se encontró en el sitio. Las mejores resistencias a heladas se observaron en urunday, especie que también presentó elevadas tolerancias a situaciones de sequias.

\section{Sitio 2}

La medición realizada en diciembre de 2004 (Cuadro $N^{\circ} 3$ ), cuando las especies tenian entre 11 y 13 años, mostró que el timbó (con 13 años) presentó los mayores diámetros, pero las menores alturas y la menor supervivencia. El lapacho negro (con 11 años) le sigue en diámetro. El guatambú blanco (con 13 años) presentó menor diámetro, pero la misma altura y supervivencia que el lapacho. Se efectuaron podas anuales de corrección del fuste para el timbó y lapacho. En guatambú se realizaron solamente algunas podas de corrección del fuste ya que su crecimiento es marcadamente monopódico. Todas las especies debieron ser protegidas contra las heladas por uno a dos inviernos, luego de 
la plantación, hasta que lograron resistencia natural con la altura (aproximadamente a los 1,5 metros, altura a la que además de una mayor resistencia de la planta, también las temperaturas minimas son mayores).

\section{Cuadro $\mathrm{N}^{\circ} 3$}

CRECIMIENTO EN DIÁMETRO, ALTURA Y SOBREVIVENCIAS DE TRES ESPECIES FORESTALES NATIVAS EN COMBINACIONES AGROFORESTALES

\begin{tabular}{|l|c|c|c|}
\hline \multicolumn{1}{|c|}{ ESPECIE } & DIÁMETRO $(\mathbf{c m})$ & ALTURA $(\mathbf{m})$ & SUPERVIVENCIA (\%) \\
\hline Enterolobium contortisiliquum & $19,3 \mathrm{a}$ & $7,92 \mathrm{a}$ & $46 \mathrm{~b}$ \\
\hline Balfourodendron riedelianum & $9,85 \mathrm{c}$ & $8,2 \mathrm{a}$ & $92 \mathrm{a}$ \\
\hline Tabebuia heptaphylla & $14,9 \mathrm{~b}$ & $7,9 \mathrm{a}$ & $90 \mathrm{a}$ \\
\hline
\end{tabular}

Sitio 2. Mediciones diciembre de 2004. Letras diferentes indican diferencias significativas entre grupos.

(Fuente: Montagnini et al., 2006b)

\section{Sitio 3}

La medición realizada en diciembre de 2004 (Cuadro $N^{\circ} 4$ ), mostró que el guatambú (a los 12 años) presentó la mayor altura y supervivencia, mientras que el timbó tuvo mayor diámetro. Se efectuaron podas de corrección del fuste para el timbó y guatambú. El guatambú debió ser apuntalado durante los primeros años para evitar la caida por viento debido a su rápido crecimiento. Ambas especies debieron ser protegidas contra las heladas por uno a dos inviernos siguientes a la plantación.

\section{Cuadro $\mathrm{N}^{\circ} 4$ \\ PROMEDIOS EN DIÁMETRO, ALTURA Y SOBREVIVENCIA PARA DOS ESPECIES FORESTALES NATIVAS EN COMBINACIONES AGROFORESTALES}

\begin{tabular}{|c|c|c|c|}
\hline ESPECIE & DIAMETRO $(\mathrm{cm})$ & ALTURA $(\mathrm{m})$ & SUPERVIVENCIA $(\%)$ \\
\hline Enterolobium contortisiliquum & $16,9 \mathrm{a}$ & $7,7 \mathrm{~b}$ & $62 \mathrm{~b}$ \\
\hline Balfourodendron riedelianum & $15,7 \mathrm{~b}$ & $11,3 \mathrm{a}$ & $75 \mathrm{a}$ \\
\hline
\end{tabular}

Sitio 3. Mediciones diciembre de 2004. Letras diferentes indican diferencias significativas entre grupos.

(Fuente: Montagnini et al., 2006b)

\section{- Discusión}

El resultado más importante en términos de crecimiento de los árboles es el excelente comportamiento de $B$. riedelianum, una de las especies más apreciadas de la región (Eibl et al., 1998). Aparentemente esta especie crece bien en buenas condiciones de suelos (fertilidad y manejo adecuado), como se observa por las mejores alturas alcanzadas en el Sitio 3, mientras que en el Sitio 1, esta especie no se adaptó a pesar de realizarse reposiciones por un total de tres años, debido a la alta degradación de este sitio. En el Sitio 2, esta especie creció pobremente 
durante el primer par de años, posiblemente debido a la baja fertilidad del suelo. Sin embargo, pasada la etapa crítica del establecimiento, esta especie presentó un buen crecimiento en suelos con condiciones nutricionales pobres. Los niveles de crecimiento mostrados para el Sitio 3 de Santiago de Liniers superan los encontrados en otras experiencias en la región donde $B$. riedelianum ha sido ensayado en plantaciones de enriquecimiento (Montagnini et al., 1997).

E. contortisiliquum se destacó por tener el mejor crecimiento en el sitio más degradado (Sitio 1), sugiriendo su utilización para recuperación de suelos pobres. En una investigación realizada cuando los árboles tenían 9 años se encontró mayor contenido de nitrógeno en el suelo superficial bajo esta especie que en las parcelas testigo con pastos (Méndez y Steitzer 1999). La susceptibilidad del E. contortisiliquum al ataque de Epicauta adspersa ("bicho moro") sugirió dudas acerca de la posibilidad de establecer esta especie en condiciones de plantación a cielo abierto. Sin embargo los ataques fueron intensos únicamente para el caso del sitio de menor degradación y solamente afectaron a los árboles en sus estadíos iniciales. En otros experimentos de la región, $E$. contortisiliquum presenta buen crecimiento en ensayos de enriquecimiento (Montagnini et al., 1997).

EI T. heptaphylla, para el Sitio 2 manifestó un buen crecimiento ya que aún plantado dos años después superó a $B$. riedelianum en diámetro y la igualó en altura. La elevada mortandad de árboles de $A$. balansae y $P$. dubium en el Sitio 1 pudo estar explicada por la compactación del suelo, que no permitió que las raíces exploraran en profundidad para el abastecimiento de agua ante condiciones de sequia. Ambas especies presentaron rebrote de cepa de aproximadamente el $20 \%$ de los ejemplares muertos en pie, con fustes de 1,5 metros en promedio al año. Esto sugiere la posibilidad de conducción de ambas especies a partir del rebrote de cepa. En el Sitio 2 las plantaciones en asociación con I. paraguariensis no afectaron las producción de hoja verde en función a los datos de peso seco de la misma al momento de la cosecha (Eibl et al., 2000).

Las plantaciones mixtas tuvieron beneficios en el Sitio 1 para todas las especies a excepción del $L$. muehlbergianum. El beneficio de las plantaciones mixtas para $E$. contortisiliquum y $A$. balansae fue de una mayor supervivencia, mientras que para $P$. dubium, la plantación mixta resultó en un mayor diámetro y altura. Otra ventaja de las plantaciones mixtas es su mayor diversidad, con el consecuente beneficio para la restauración de las propiedades del ecosistema.

\section{ENRIQUECIMIENTO DE BOSQUES DEGRADADOS}

\section{Materiales y Métodos}

\section{- Sitio del Estudio}

Los ensayos en estudio se encuentran en la Reserva Guarani, región de la Selva Subtropical Oriental (Provincia Paranaense) ubicada al NE de Misiones. Esta reserva de más de 5000 ha es manejada por la Facultad de Ciencias Forestales de la UNAM con fines productivos y de investigación y docencia. El área basal del bosque sobre-explotado fue menor de $10 \mathrm{~m}^{2 /}$ ha. El clima corresponde al mismo tipo Cfa descrito anteriormente. Los suelos son Ultisoles, 
gran grupo kandiudultes (US Soil Taxonomy), conocidos regionalmente como tierra colorada o suelo rojo profundo. Son arcillosos, con predominancia de sesquióxidos y caolinita, ácidos ( $\mathrm{pH}$ en agua 5 - 5.5), bien drenados, bien estructurados, profundos, con materia orgánica en superficie generalmente superior a 3\%, CIP (Capacidad de Intercambio Catiónico) de 10-20 $\mathrm{cmol} / \mathrm{kg}$ y porcentaje de saturación de bases mayores del $50 \%$.

\section{- Especies Ensayadas}

Se realizó enriquecimiento en fajas con el objetivo de mejorar la composición de la masa arbórea en calidad y cantidad de ejemplares, seleccionando especies que por su potencialidad de crecimiento permitieran un aprovechamiento a corto y mediano plazo (de 15 a 40 años) (Montagnini et al., 1997).

Las especies ensayadas fueron:

Balfourodendron riedelianum

Bastardiopsis densiflora

Cordia trichotoma

Ocotea puberula.

\section{Resultados}

Las especies con mejores promedios de altura y DAP a los 13 años fueron Ocotea puberula, Bastardiopsis densiflora, y Cordia trichotoma (Cuadro $\mathrm{N}^{\circ} 5$ ). Ocotea puberula, especie maderable muy apreciada, habia sufrido severos ataques de un coleóptero barrenador, Oncideres gutturator Fabr. (Cerambycidae), localmente conocido como "quiritó", "cortapalos", "taladro podador", pero luego de estos ataques los ejemplares de Ocotea puberula pudieron rebrotar vigorosamente resultando con los mejores crecimientos de las cuatro especies ensayadas. Le siguieron en crecimiento en altura Bastardiopsis densiflora y Cordia tricotoma.

\section{Cuadro $\mathrm{N}^{\circ} 5$}

PROMEDIOS DE ALTURA, DIÁMETRO Y SUPERVIVENCIA POR ESPECIE PARA LAS FAJAS DE ENRIQUECIMIENTOS EN GUARANI

\begin{tabular}{|l|c|c|c|}
\hline \multicolumn{1}{|c|}{ ESPECIE } & DIAMETRO (cm) & ALTURA (m) & SUPERVIVNCIA (\%) \\
\hline $\begin{array}{l}\text { Balfourodendron } \\
\text { riedelianum }\end{array}$ & $4,3 \mathrm{c}$ & $5,9 \mathrm{c}$ & $75 \mathrm{a}$ \\
\hline Bastardiopsis densiflora & $13,3 \mathrm{~b}$ & $10,4 \mathrm{a}$ & $65 \mathrm{~b}$ \\
\hline Ocotea puberula & $19,1 \mathrm{a}$ & $9,9 \mathrm{a}$ & $75 \mathrm{a}$ \\
\hline Cordia trichotoma & $10,9 \mathrm{~b}$ & $8,6 \mathrm{~b}$ & $75 \mathrm{a}$ \\
\hline
\end{tabular}

Ensayo instalado en 1991. Datos para el año 2004.

Letras diferentes indican diferencias significativas entre grupos

(Fuente: Montagnıni et al., 2006b) 


\section{Discusión}

Los ensayos de Guarani destacan el comportamiento superior de Ocotea puberula y Bastardiopsis densiflora en condiciones de enriquecimiento. Bastardiopsis densiflora crece bien en bosques secundarios formando rodales densos. Su madera es apreciada por los mercados locales. En experimentos previos sobre el impacto de especies forestales nativas sobre los suelos, las mayores concentraciones de carbono y nitrógeno se encontraron bajo la copa de rodales de esta especie, donde fueron el doble de lo que se encontraba fuera del área de influencia de la copa (Fernández et al., 1997). El pH del suelo asimismo fue superior bajo Bastardiopsis densiflora y Cordia trichotoma mientras que la suma de bases $\left(\mathrm{Ca}^{++} \mathrm{Mg}^{++} \mathrm{K}^{+}\right)$fue mayor bajo Cordia trichotoma, Bastardiopsis densiflora y Enterolobium contortisiliquum. De esta manera, estas especies que tienen buen comportamiento en enriquecimiento también pueden ser beneficiosas al mejorar las condiciones de los suelos en áreas de bosques degradados.

Entre las posibles desventajas del enriquecimiento se citan los costos elevados de apertura de fajas y del cuidado de las plantaciones en los primeros años. Además, las condiciones lumínicas pueden no ser las más favorables para las especies implantadas. Por otro lado, debido a hallarse más expuestas, aumenta la susceptibilidad de las especies implantadas al ataque de plagas. Sin embargo, el manejo del enriquecimiento puede adaptarse a las necesidades de cada bosque. El mantenimiento de la limpieza en las fajas de enriquecimiento favorece el desarrollo de la regeneración natural (Montagnini et al., 1997).

Es necesario ajustar los tratamientos silviculturales no solamente para el establecimiento de la plantación y crecimiento de las lineas sino también para el desarrollo de la regeneración natural entre las lineas. Si se considera en conjunto los ejemplares arbóreos del enriquecimiento y la regeneración natural como la masa en crecimiento para un futuro aprovechamiento, el enriquecimiento se torna una alternativa económicamente más atractiva. Además, la combinación del enriquecimiento de especies maderables con otras como el Euterpe edulis, (palmito) o especies frutales, de turno de aprovechamiento más corto, acelera el retorno de la inversión, tornando esta técnica más atractiva.

\section{CONCLUSIONES}

En plantaciones destinadas a reforestar sitios con diferentes grados de degradación, para el sitio de mayor degradación química y física, tanto Enterolobium contortisiliquum como Astronium balansae presentaron muy buena adaptación.

Enterolobium contortisiliquum se destacó más en el sitio de mayor degradación, lo cual indica que es una especie apropiada para la recuperación de sitios degradados.

Balfourodendrum riedelianum y Bastardiopsis densiflora no se adaptaron a situaciones de elevada degradación fisica y quimica. Asimismo, Lonchocarpus mueh/bergianum aparentemente es una especie no adaptada a sitios de alta degradación, desconociéndose su respuesta ante mejores condiciones de suelo. 
de crecimiento y adaptación a sitios de alta degradación. Se trata de una especie de bajos requerimientos nutricionales para un buen crecimiento, pero sensible a las condiciones de compactación por lo que se sugiere una preparación del suelo con subsolado hasta los $60 \mathrm{~cm}$ de profundidad.

En las condiciones de mediana fertilidad los mejores resultados se obtuvieron con Balfourodendron riedelianum y Tabebuia heptaphylla.

EI B. riedelianum tuvo los mejores crecimientos en el sitio de mejor fertilidad y menor compactación.

En experimentos de enriquecimiento de bosques degradados, en la Reserva Guarani, a los 13 años las mejores especies fueron Ocotea puberula, Bastardiopsis densiflora, y Cordia trichotoma. Estas especies también tienen efectos beneficiosos sobre los suelos de los bosques degradados. La incorporación de especies con cosecha más temprana tal como la palma Euterpe edulis puede acelerar el retorno de la inversión.

El enriquecimiento agrega valor a bosques sobreexplotados de bajo volumen comercial, previniedo su conversión a otros usos de la tierra.

Con el aumento de la superficie de bosques degradados y bosques secundarios a nivel mundial, el enriquecimiento puede ser un técnica cada vez más importante para la restauración de bosques degradados. En Misiones, los agricultures locales buscan información para seleccionar especies para enriquecimiento de bosques degradados y secundarios.

La Reserva Guarani se encuentra dentro de la Reserva Yaboti, de más de 250.000 ha, la cual es una Reserva de la Biósfera. A su vez, ambas reservas forman parte del "Corredor Verde ", que conecta el bosque misionero con el resto del bosque Paranaense y con la Mata Atlántica del Brasil. De manera que recomendaciones sobre especies y manejo pueden ser utilizadas para promover el buen uso y conservación de estas importantes áreas de bosque subtropical y tropical. Localmente, en la provincia de Misiones, los resultados de estas investigaciones se están utilizando para estimular la plantación de árboles en proyectos comunitarios, tanto en sistemas agroforestales o de plantaciones a cielo abierto como de enriquecimiento de bosques degradados. 


\section{REFERENCIAS}

Cozzo, D., 1969. Siete años de un ensayo de enriquecimiento del bosque subtropical utilizando Cordia trichotoma. Revista Forestal Argentina. Año XIII.n 2. Buenos Aires. Argentina. pp.44-45.

Eibl, B., Montagnini, F., Fernández, R., Kozarik, J., Nozzi, D. y Lupi, A., 1997. Sistemas agroforestales con llex paraguariensis (yerba Mate) y árboles maderables nativos en pequeñas propiedades en Misiones, Argentina.: Actas Segundo Congreso Forestal Argentino y Latinoamericano. Asociación Forestal Argentina. Del 13-15 agosto 1997, Posadas. Misiones, Argentina.. Pp. 1-9.

Eibl, B., Montagnini, F., 1998. El potencial de las especies nativas en programas de plantación. En: VI Jornadas Técnicas. Serie Técnica $n^{\circ} 6$. Ecologia de Especies Nativas de la Selva Subtropical Misionera. Instituto Subtropical de Investigaciones Forestales. Universidad Nacional de Misiones, Facultad de Ciencias Forestales. Eldorado, Misiones, Argentina. Mayo, 1998. Pp.19-26.

Eibl, B., Fernández, R., Kozarik, J., Lupi, A., Montagnini, F. and Nozzi, D., 2000. Agroforestry systems with llex paraguariensis (American holly or yerba mate) and native timber trees on small farms in Misiones, Argentina. Agroforestry Systems 48:1-8.

Eibl, B., Silva, F., Bobadilla, A., 2001. Boletin Agrometeorológico Eldorado. Instituto Subtropical de Investigaciones Forestales. Facultad de Ciencias Forestales. UNaM. Periodo 1985/2001.

Fernández, R., Montagnini, F. y Hamilton, H., 1997. The influence of native tree species on soil chemistry in a subtropical forests of the Paranaense region of Misiones, Argentina. Forest Ecology and Management. 99:237-246.

Gartland, H. M., 1974. Posibilidades de enriquecimiento del bosque subtropical misionero. Boletin Argentino Forestal. Año XXXII. No.298. pp.23-30.

Gobierno de la Provincia de Misiones, 2003. Actitud Forestal. Un mundo de oportunidades para una Misiones estratégica. Subsecretaria de Bosques y Forestaciones. Ministerio de Ecologia, Recursos Naturales y Turismo. Posadas, Misiones, Argentina.

Mangieri, H.R., 1965. Reconstitución de los bosques misioneros y caracteristicas biológicas de las principales especies. En: Primeras Jornadas de trabajo del CEBS. Eldorado - Misiones. p. 141-145.

Méndez, R. y Steitzer, E., 1999. Influencia de una especie fijadora de nitrógeno, Enterolobium contortisiliquum (Vell) Morong (timbó) sobre la recuperación de un suelo degradado. Informe Final de Proyecto Integradora I. Facultad de Ciencias Forestales. Universidad Nacional de Misiones. Eldorado. Misiones. Pp. 17.

Ministerio de Ecologia, Recursos Naturales Renovables y Turismo, 2003. Estrategias de desarrollo desde el sur forestal mundial. Plan Maestro Forestal. Memoria 2000 - 2003 y Perspectiva del patrimonio forestal industrial. Formato CD. Gobierno de la Provincia de Misiones. 
Posadas. Misiones. Argentina.

Montagnini, F., Fernández, R. y Hamilton, H., 1995. Relación entre especies nativas y la fertilidad de los suelos. Parte 1: Contenido de elementos en la biomasa. Yvyraretá (Argentina) 6(6): 5-12.

Montagnini, F., Eibl, B., Grance, L., Maiocco, D. and Nozzi, D., 1997. Enrichment planting in degraded forests of the Paranaense region of Misiones, Argentina. Forest Ecology and Management 99 (1 y 2): 237-246.

Montagnini, F., Eibl, B. y Fernández, R., 2006a. Adaptabilidad y crecimiento de especies forestales nativas de bosque húmedo subtropical en sitios degradados. Yvyraretá (Argentina) 15:10-16.

Montagnini, F., Eibl, B. y Fernandez, R., 2006b. Rehabilitation of degraded lands in Misiones, Argentina. Bois et Forets des Tropiques 288: 51-65.

Sánchez, J. R., Gotz, I. y Segovia, W., 1993. Enriquecimiento de bosques nativos. Implantación bajo cubierta. Tercera comunicación. En: VII Jornadas Técnicas: Ecosistemas Forestales Nativos. Uso, Manejo y Conservación. Facultad de Ciencias Forestales. Eldorado. Misiones. p. 300-308. 
